\title{
The focal geometry of circular and conical meshes
}

\begin{abstract}
Circular meshes are quadrilateral meshes all of whose faces possess a circumcircle, whereas conical meshes are planar quadrilateral meshes where the faces which meet in a vertex are tangent to a right circular cone. Both are amenable to geometric modeling - recently surface approximation and subdivision-like refinement processes have been studied. In this paper we extend the original defining property of conical meshes, namely the existence of face/face offset meshes at constant distance, to circular meshes. We study the close relation between circular and conical meshes, their vertex/vertex and face/face offsets, as well as their discrete normals and focal meshes. In particular we show how to construct a two-parameter family of circular (resp., conical) meshes from a given conical (resp., circular) mesh. We further discuss meshes which have both properties and their relation to discrete surfaces of negative Gaussian curvature.

The offset properties of special quadrilateral meshes and the threedimensional support structures derived from them are highly relevant for computational architectural design of freeform structures. Another aspect important for design is that both circular and conical meshes provide a discretization of the principal curvature lines of a smooth surface, so the mesh polylines represent principal features of the surface described by the mesh.
\end{abstract}

2000 Mathematics Subject Classification: 68U05, 53A40, 52C99, $51 B 15$

Keywords discrete differential geometry, architectural design, geometric modeling, integrable systems, quadrilateral meshes, conical meshes, circular meshes, offset meshes, focal meshes.

Helmut Pottmann, Johannes Wallner

Institut für Diskrete Mathematik und Geometrie, Technische Universität Wien

Wiedner Hauptstr. 8-10/104, A 1040 Wien, Austria

E-mail: pottmann@geometrie.tuwien.ac.at 


\section{Introduction}

Circular meshes are quadrilateral meshes all of whose faces possess a circumcircle, whereas conical meshes are planar quadrilateral meshes where the faces which meet in a vertex are tangent to a right circular cone. Both the circular and the conical meshes are discrete counterparts of the network of principal curvature lines of a smooth surface. It turns out that for both discretizations, important properties known in the smooth case carry over to the discrete setting. This is especially true for properties of the focal surfaces, and the developable surfaces formed by the surface normals along principal curvature lines.

The natural geometric setting for circular meshes is Möbius geometry. Conical meshes are their Laguerre geometry counterpart. The present paper however is concerned with purely Euclidean properties of such meshes - their discrete normals, offsets and focal surfaces.

We study the geometry of circular and conical meshes, parallel meshes inscribed or circumscribed to the unit sphere, the system of discrete normals, and the close relation between circular and conical meshes. Special emphasis is laid on offset meshes of constant face and constant vertex distance. We also discuss meshes which are both circular and conical. Among those there is a class of meshes derived from a well known discretization of surfaces of constant negative curvature.

\subsection{Previous work}

The geometry of quadrilateral meshes with planar faces ( $P Q$ meshes) has been studied within the framework of difference geometry, which is one of the precursors of discrete differential geometry $[6,10]$. It has been observed that such meshes are a discrete counterpart of conjugate curve networks on smooth surfaces. Earlier contributions are found in the work of R. Sauer from 1930 onwards, culminating in his monograph [24]. Recent contributions, especially on the higher-dimensional case, include the work of Doliwa, Santini and Mañas $[13,14,18]$. In the literature PQ meshes are sometimes called simply quadrilateral meshes.

The interesting case of a circular mesh where all quads possess a circumcircle has been introduced by Martin et al. [19]. It is known to be a discrete analogue of the network of principal curvature lines. Pointers to the literature on PQ meshes and circular meshes are given on pp. 39 and 46 of [6]. Some papers dealing with circular meshes are the following ones: A discretization of triply orthogonal systems was proposed by Bobenko [2]. In Cieslinski et al. [9], discrete orthogonal nets were generalized to arbitrary dimensions. Bobenko et al. [4] studied discrete minimal surfaces represented by circular meshes. Conical meshes, their computation, and their properties relevant for architectural design are the topic of [17]. It is not difficult to see that the discrete representations of surfaces with constant negative Gaussian curvature studied by W. Wunderlich and R. Sauer $[23,27]$ can easily be converted into PQ meshes, which are both circular and conical. For further related developments, see e.g. $[3,12,16]$. Very recently, 

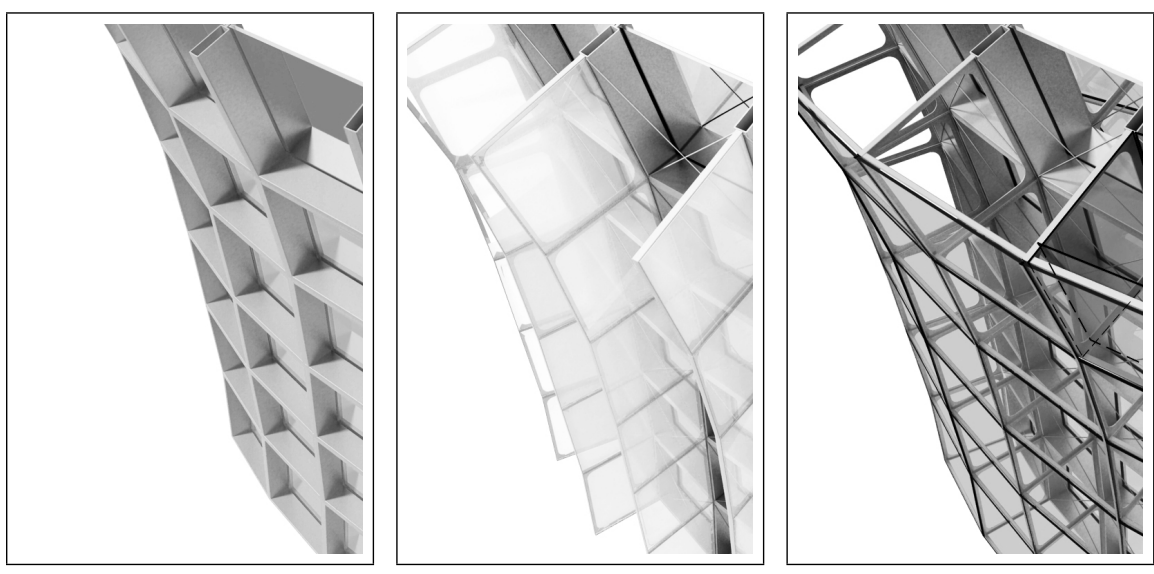

Fig. 1 A conical mesh possesses face/face offset meshes at constant distance, and can be assigned discrete normals in the vertices, which connect the vertices of the mesh with the vertices of the offset mesh, and which form a 3D support structure all of whose quadrilaterals are planar. This fact is relevant in the architectural design of freeform glass structures. Images: B. Schneider.

circular and conical meshes have been treated together from the unifying viewpoint of Lie sphere geometry [7].

\subsection{Applications}

The offset properties of special quadrilateral meshes and the three-dimensional support structures derived from them are highly relevant for computational architectural design of freeform structures. Another aspect important for design is that both circular and conical meshes provide a discretization of the principal curvature lines of a smooth surface, so the mesh polylines represent principal features of the surface described. We would like to briefly demonstrate these applications of conical meshes in building construction and architectural design, which are the topic of [17]. Fig. 1 shows a multilayered construction based on a conical mesh, its offsets, and the discrete surface normals. Fig. 2 shows the result of a combination of subdivision and mesh optimization according to [17].

\section{Definitions and notation}

A mapping $\mathbf{v}: \mathbb{Z}^{2} \rightarrow \mathbb{R}^{d}$ is called a quadrilateral mesh. We usually write $\mathbf{v}_{i j}$ instead of $\mathbf{v}(i, j)$, where $(i, j) \in \mathbb{Z}^{2}$. For simplicity, we consider a mesh to be defined over all of $\mathbb{Z}^{2}$. We usually study the case $d=3$, but later also consider $d=4$. We consider, for each index $(i, j) \in \mathbb{Z}^{2}$ the elementary quadrilateral with vertices $\mathbf{v}_{i, j}, \mathbf{v}_{i-1, j}, \mathbf{v}_{i-1, j-1}$, and $\mathbf{v}_{i, j-1}$.

A mesh is a PQ mesh, if all elementary quadrilaterals span a 2-dimensional affine subspace $F_{i j}$, i.e., a plane. This plane is called the face plane. 

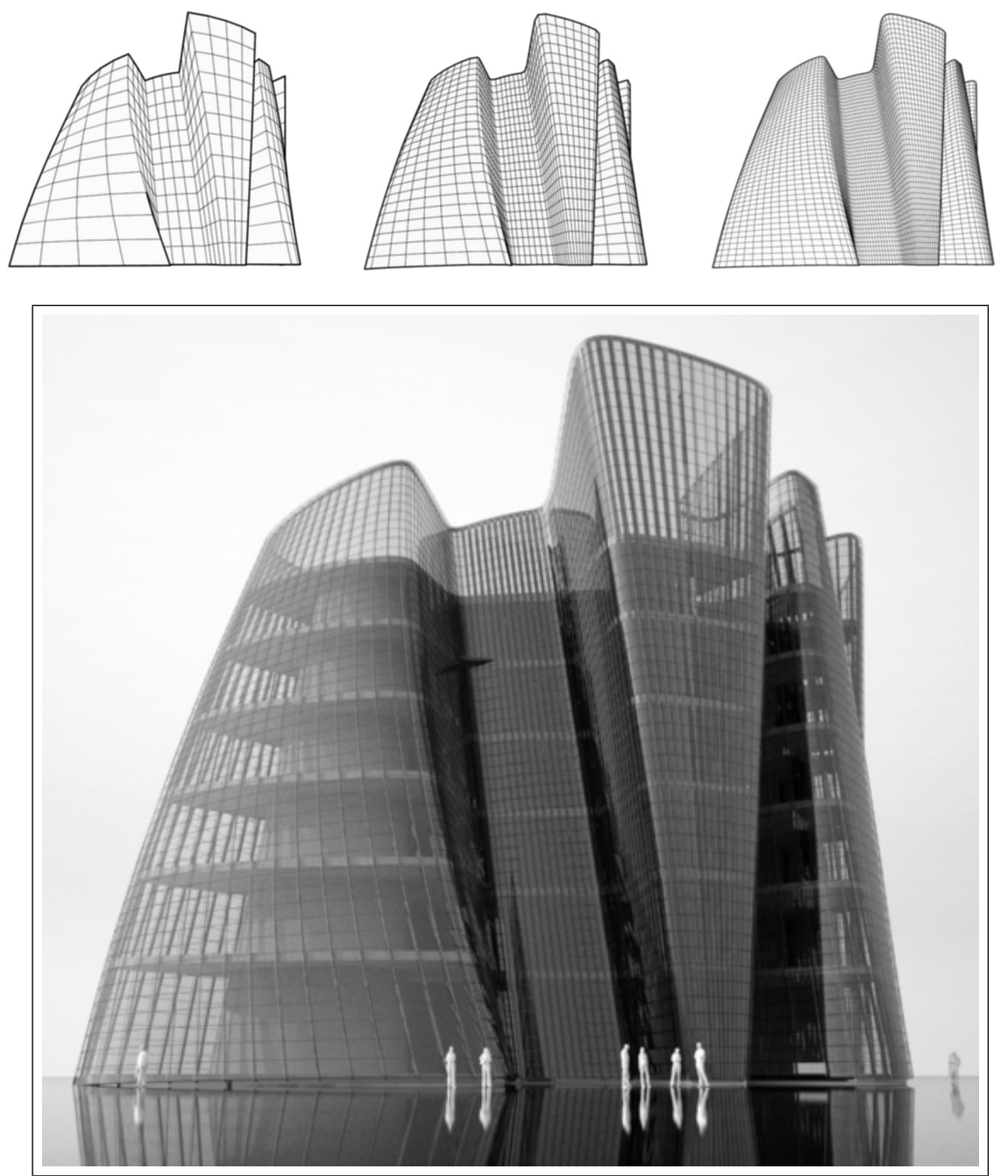

Fig. 2 A sequence of conical meshes produced by subdivision and mesh optimization according to [17], which is the basis of an incomplete, especially roofless, architectural design. Images: B. Schneider.

The vertex $\mathbf{v}_{i j}$ is contained in the planes $F_{i, j}, F_{i+1, j}, F_{i+1, j+1}$, and $F_{i, j+1}$, We denote by $\mathcal{P}$ the set of oriented planes of Euclidean $\mathbb{R}^{3}$. The mapping $F: \mathbb{Z}^{2} \rightarrow \mathcal{P}$ is a plane-valued mesh dual to the original one. An oriented plane is determined by its unit normal vector $\mathbf{n}=\left(n_{1}, n_{2}, n_{3}\right) \in S^{2}$ and the absolute coefficient $n_{0}$ in the equation of the plane: $n_{0}+\langle\mathbf{n}, \mathbf{x}\rangle=0$. When we use these coordinates, $\mathcal{P}$ becomes a subset of $\mathbb{R}^{4}$, and $F$ can also be seen as an $\mathbb{R}^{4}$-valued mesh.

The mesh $\mathbf{v}$ is circular, if all elementary quadrilaterals possess a circumcircle. It is conical, if for all vertices $\mathbf{v}_{i j}$ there is an oriented sphere, such that 

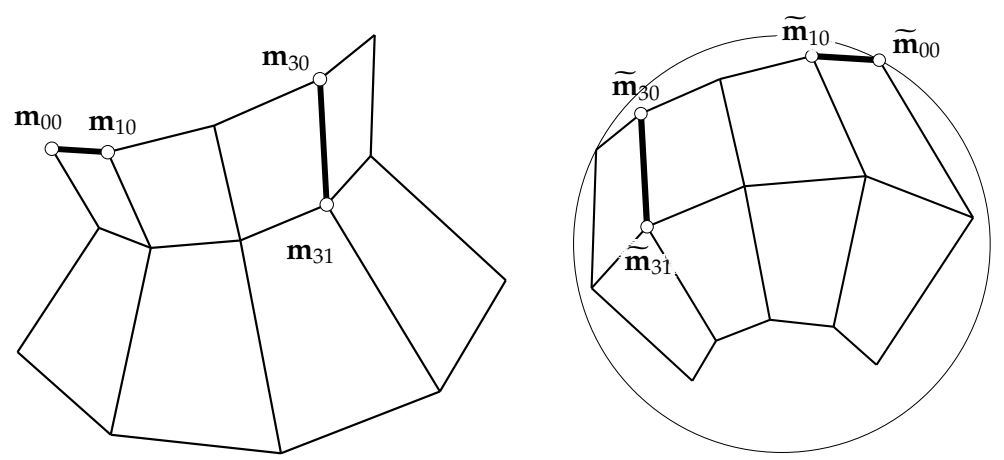

Fig. 3 Two meshes which are Combescure transforms of each other: $\mathbf{m}$ is a circular mesh, and $\widetilde{\mathbf{m}}$ is a parallel mesh whose vertices are contained in the unit sphere.

the oriented planes incident with $\mathbf{v}_{i j}$ are tangent to that sphere. In coordinates, this means the following: The oriented plane $\left(n_{0}, \mathbf{n}\right) \in \mathcal{P}$ and the oriented sphere $(r, \mathbf{m})$ with center $\mathbf{m} \in \mathbb{R}^{3}$ and radius $r \in \mathbb{R}$ are in oriented contact, if and only if $n_{0}+\langle\mathbf{n}, \mathbf{m}\rangle=r$. In order to avoid degenerate cases, the definition of conical mesh requires that the sphere mentioned there must not have zero radius, so without loss of generality we may assume that the radius equals 1 . Planes which pass through a point and are in addition tangent to a sphere are also tangent to a cone of revolution, which explains the name conical. The axis of that cone is the line spanned by the vertex and the center of the sphere.

Quadrilaterals $\mathbf{a}_{1}, \ldots, \mathbf{a}_{4}$ and $\mathbf{a}_{1}^{\prime}, \ldots, \mathbf{a}_{4}^{\prime}$ are called parallel, if corresponding edges are parallel. Meshes $\mathbf{v}, \mathbf{w}$ are Combescure transforms of each other, or are parallel meshes, if corresponding quads are parallel. We could also define this by requiring that corresponding edges are parallel. PQ meshes are face offsets of each other, if they are parallel and their faces are at constant oriented distance. They are vertex offsets of each other, if they are parallel meshes, but no translates of each other, and their vertices are at constant nonzero oriented distance. All concepts defined here are well known, except the conical meshes which have been considered first in [17].

\section{Geometry of conical and circular meshes}

\subsection{Conical meshes}

Conical meshes have been introduced by Liu et al. [17]. It has been shown [17] and in [26] that meshes which have nontrivial face offsets are conical, and that translating the face planes of a conical mesh by a fixed distance in the direction of normal vectors yields face offsets. This fact is employed in [17] in applications of conical meshes to architectural design.

The computation of conical meshes according to [17] is based on an optimization algorithm which perturbs a PQ mesh such that it becomes conical. The conical property at vertices can be expressed in terms of the 

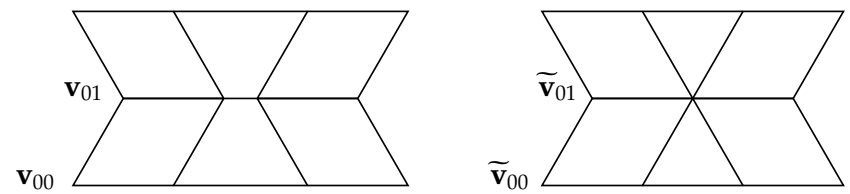

Fig. 4 Two parallel quad meshes $\mathbf{v}$ and $\widetilde{\mathbf{v}}$, such that one of them contains a zero edge.

successive four angles $\omega_{1}, \ldots, \omega_{4}$ enclosed by the four edges emanating from that vertex:

Proposition 1 A quad mesh is conical if and only if for all vertices, the four interior angles $\omega_{1}, \ldots, \omega_{4}$ successively enclosed by the edges emanating from that vertex obey $\omega_{1}+\omega_{3}=\omega_{2}+\omega_{4}$.

A proof is given in [26]. For the following result concerning parallel meshes of conical meshes, we consider the unit sphere to be oriented such that normal vectors point outwards. If we choose the origin of the coordinate system as center, and radius 1 , this is consistent with the previous definition of oriented contact.

Proposition 2 For each conical mesh $\mathbf{v}$ there is a unique conical mesh $\widetilde{\mathbf{v}}$ parallel to $\mathbf{v}$ whose faces are tangent to the unit sphere (provided adjacent faces are not co-planar; it may happen that certain edges become zero).

Proof Consider the faces $F: \mathbb{Z}^{2} \rightarrow \mathcal{P}$ of the given mesh. For each oriented plane $F_{i j}$ there is an oriented plane $\widetilde{F}_{i j}$ tangent to the unit sphere. The 4-tuple of faces $F_{i j}, F_{i+1, j}, F_{i+1, j+1}, F_{i, j+1}$, incident with $\mathbf{v}_{i j}$ and the corresponding 4tuple $\widetilde{F}_{i j}, \widetilde{F}_{i+1, j}, \widetilde{F}_{i+1, j+1}, \widetilde{F}_{i, j+1}$ are parallel translates of each other, both being tangent to a sphere of radius 1 . Consequently these four planes intersect in a common vertex $\widetilde{\mathbf{v}}_{i j}$. Because of the parallelity of corresponding faces, the meshes $\mathbf{v}$ and $\widetilde{\mathbf{v}}$ have parallel corresponding edges (here we have used that adjacent faces are not co-planar).

The construction of Prop. 2 does not check if the vertices of $\widetilde{\mathbf{v}}$ are all different. It may happen that zero edges occur, as illustrated by Fig. 4. One example of this is the following: If the face planes $F_{00}, F_{10}, F_{20}, F_{01}, F_{11}, F_{21}$ of a conical mesh happen to have unit normal vectors which are contained in a circle of the unit sphere $S^{2}$, then the faces $\widetilde{F}_{i j}$ which are parallel to $F_{i j}$ but tangent to $S^{2}$ have a point in common, as visualized by Fig. 4 , right.

\subsection{Circular meshes}

In some respects, properties of circular meshes are similar or dual to properties of conical meshes. It is therefore to be expected that circular meshes possess a parallel mesh which is closely associated with the unit sphere, just as the conical meshes do. It turns out that this is true, but without the 
uniqueness we encountered in the conical case. A circular mesh always has a vertex offset, which means a parallel mesh at constant vertex-vertex distance. Of course, this can be achieved in a trivial way by simply translating the given mesh by a fixed vector. We are are however interested in nontrivial vertex offsets, which are not constructed in this way, i.e., are not simply translates of each other.

Section 3.2 is structured as follows. First, Lemma 3 recalls a well known property of quadrangles. Then Proposition 4 shows that all circular meshes have parallel meshes inscribed into the unit sphere. These auxiliary meshes are used by Lemma 7 to construct a family of offsets at constant vertexvertex distance. Finally, Theorem 8 states that this property characterizes circular meshes.

Lemma 3 If a quadrilateral has a circumcircle, then this is true for every parallel quadrilateral (in the case of coincident vertices, their affine span, which is used for testing for parallelity of edges, is replaced by the tangent of the circumcircle).

The following proposition has been shown in [16, p. 3084] in a more general context. For the convenience of the reader, we include an elementary proof. This result has nice consequences, which seem to be new.

Proposition 4 Any circular mesh $\mathbf{v}$ has a parallel mesh $\widetilde{\mathbf{v}}: \mathbb{Z}^{2} \rightarrow S^{2}$ whose vertices are contained in the unit sphere. One vertex, say $\widetilde{\mathbf{v}}_{00}$, can be chosen arbitrarily, and the mesh $\widetilde{\mathbf{v}}$ is circular (zero edges may occur).

Conversely, any $P Q$ mesh $\mathbf{v}$ which has a nontrivial parallel mesh $\widetilde{\mathbf{v}}$ in the unit sphere, is a circular mesh.

Proof A line which intersects the sphere $S^{2}$ in a point has a unique second intersection point which is different from the first one if and only if the line is not tangent to $S^{2}$. It follows that from $\widetilde{\mathbf{v}}_{00}$ we can successively construct $\widetilde{\mathbf{v}}_{i 0}$ and $\widetilde{\mathbf{v}}_{0 j}$ for all $i, j \in \mathbb{Z}$. As to other indices, it is obviously sufficient to show how to construct $\widetilde{\mathbf{v}}_{i+1, j+1}$ from the three points $\widetilde{\mathbf{v}}_{i j}, \widetilde{\mathbf{v}}_{i+1, j}$, and $\widetilde{\mathbf{v}}_{i, j+1}$ : We intersect the line through $\widetilde{\mathbf{v}}_{i+1, j}$ parallel to $\mathbf{v}_{i+1, j+1}-\mathbf{v}_{i+1, j}$ and the line through $\widetilde{\mathbf{v}}_{i, j+1}$ parallel to $\mathbf{v}_{i+1, j+1}-\mathbf{v}_{i, j+1}$. It follows from Lemma 3 that the elementary quadrilateral $\widetilde{\mathbf{v}}_{i j}, \ldots$ has a circumcircle, then contained in $S^{2}$. This implies that $\widetilde{\mathbf{v}}_{i+1, j+1} \in S^{2}$.

The converse is easy to show: Any $P Q$ mesh in the unit sphere is obviously circular, and by Lemma 3 so are its parallel meshes.

Remark 5 The construction of the previous proof can result in zero edges, i.e., coincident vertices. The result remains valid if we replace the affine span of two coincident vertices, which is used for testing for parallelity, by the common tangent of the circumcircles which pass through this point.

Remark 6 The construction of a discrete minimal surface from an isothermic mesh $\mathbf{v}: \mathbb{Z}^{2} \rightarrow S^{2}$ via a discrete Christoffel duality as described in [5] is an inverse of the construction of Proposition 4.

The following result together with Theorem 8 describes the existence and construction of vertex offsets. 
Lemma 7 A circular mesh $\mathbf{v}: \mathbb{Z}^{2} \rightarrow \mathbb{R}^{3}$ has a family of parallel circular meshes $\mathbf{v}^{(r)}(r \in \mathbb{R})$ which are no translates of $\mathbf{v}$, such that $\left\|\mathbf{v}_{i j}^{(r)}-\mathbf{v}_{i j}^{(s)}\right\|=|r-s|$, independent of indices $i$ and $j$.

Proof Find a parallel mesh $\widetilde{\mathbf{v}}: \mathbb{Z}^{2} \rightarrow S^{2}$ and let $\mathbf{v}^{(r)}:=\mathbf{v}+\widetilde{r}$. By construction, the mesh $\mathbf{v}^{(r)}$ is parallel to $\mathbf{v}$, and $\mathbf{v}^{(r)}-\mathbf{v}^{(s)}=(r-s) \widetilde{\mathbf{v}}$, with $\|\widetilde{\mathbf{v}}\|=$ const $=1$. As $\widetilde{\mathbf{v}}$ is not the constant mesh, the meshes constructed here are no translates of $\mathbf{v}$

Note that the parallel meshes constructed here rely on the meshes $\widetilde{\mathbf{v}}$ of Proposition 4, which are not unique.

Theorem 8 The $P Q$ mesh $\mathbf{v}$ is circular if and only if it has a vertex offset $\mathbf{v}^{\prime}$ at distance $d \neq 0$.

Proof The existence of $\mathbf{v}^{\prime}$ follows from the previous theorem with $r=0$ and $s=1$. Starting with $\mathbf{v}^{\prime}$, we let $\widetilde{\mathbf{v}}=\left(\mathbf{v}^{\prime}-\mathbf{v}\right) / d$. By construction, the mesh $\widetilde{\mathbf{v}}$ is parallel to $\mathbf{v}$, and is contained in the unit sphere. Our assumption on $\mathbf{v}^{\prime}$ implies that $\widetilde{\mathbf{v}}$ is not a constant mesh. It now follows from Proposition 4 that $\mathbf{v}$ is circular.

These properties of circular meshes show that also circular meshes are interesting for applications, e.g. in architectural design. We should mention at this point that the offset meshes used in the previous constructions may look like surfaces with an edge of regression and have self-intersections as their distance from the original mesh increases. This however does not destroy our arguments.

\subsection{The Gaussian image}

Differential geometry employs the Gaussian image of a smooth surface, which is the mapping of that surface to the unit sphere via its unit normal vectors. In the context of circular and conical meshes, there are two obvious candidates for a discrete version of this concept. Meshes are always oriented.

Definition 9 For a conical mesh $\mathbf{v}$, consider the unit vectors $\mathbf{n}_{i j}$ which indicate the direction of the cone axis at the vertex $\mathbf{v}_{i j}$. For a circular mesh $\mathbf{v}$, consider the unit normal vectors $\mathbf{n}_{i j}$ of the face planes $F_{i j}$. In both cases, the mesh $\mathbf{n}: \mathbb{Z}^{2} \rightarrow S^{2}$ is called the Gaussian image of $\mathbf{v}$.

We show a relation between the Gaussian image of a circular mesh and its spherical Combescure transforms. In order to distinguish between subspaces in the sense of affine geometry (i.e., points, lines and planes), and subspaces in the sense of linear algebra (all of which pass through the origin), we use the term 'linear subspace' for the latter.

Lemma $\mathbf{1 0}$ For any circular mesh $\mathbf{v}: \mathbb{Z}^{2} \rightarrow \mathbb{R}^{3}$, consider the Gaussian image mesh $\mathbf{n}: \mathbb{Z}^{2} \rightarrow S^{2}$ and a parallel mesh $\widetilde{\mathbf{v}}: \mathbb{Z}^{2} \rightarrow S^{2}$. Then the mesh $\mathbf{n}$ consists of the spherical centers of the circumcircles of $\widetilde{\mathbf{v}}$, and adjacent vertices of $\widetilde{\mathbf{v}}$ lie symmetric with respect to the linear subspaces spanned by adjacent vertices of $\mathbf{n}$. 
Proof The faces of $\widetilde{\mathbf{v}}$ and of $\mathbf{v}$ are parallel, therefore so are the axes of circumcircles, and the statement about centers of circumcircles follows. Now consider two adjacent vertices of $\widetilde{\mathbf{v}}$, say $\widetilde{\mathbf{v}}_{i j}$ and $\widetilde{\mathbf{v}}_{i+1, j}$. They are contained in two circumcircles: one with center $\mathbf{n}_{i j}$, and one with center $\mathbf{n}_{i, j-1}$. The two intersection points of these circumcircles lie symmetric with respect to the subspace spanned by the vectors $\mathbf{n}_{i j}, \mathbf{n}_{i, j-1}$.

Remark 11 Lemma 10 yields another way of constructing the spherical mesh $\widetilde{\mathbf{v}}$ parallel to a given circular mesh $\mathbf{v}$, once a vertex $\widetilde{\mathbf{v}}_{00}$ is chosen: We successively apply reflections in the subspaces spanned by the origin and the edges of the Gaussian image mesh.

\section{Möbius and Laguerre geometry}

We first briefly review the connection between circular meshes in $\mathbb{R}^{3}$ and Möbius geometry. For a more detailed treatment of Möbius geometry the interested reader is referred to [15]. Three-dimensional Möbius space $\mathbb{R}^{3} \cup\{\infty\}$ consists of Euclidean 3-space plus a point at infinity. Its standard point model is the sphere $S^{3}$. The passage from $S^{3}$ to $\mathbb{R}^{3}$ is achieved by a stereographic projection from the point $(0,0,0,1)$ onto the equator 3 -space. The north pole thereby is mapped to the point at infinity. Circles of $\mathbb{R}^{3}$ are represented as circles in $S^{3}$ which do not pass through the north pole, whereas the straight lines of $\mathbb{R}^{3}$ correspond to those circles of $S^{3}$ which contain the north pole. If we assume that such circles do not occur, there is an obvious bijection between the circular meshes in $\mathbb{R}^{3}$ and the circular meshes in $S^{3}$. $S^{3}$ itself would be a more natural geometry setting for the discussion of circular meshes. Any planar quadrilateral whose vertices lie in $S^{3}$ has a circumcircle, so the set of circular meshes $\mathbf{v}: \mathbb{Z}^{2} \rightarrow S^{3}$ coincides with the set of planar quadrilateral meshes $\mathbf{v}: \mathbb{Z}^{2} \rightarrow S^{3}$.

A similar relation is true for conical meshes and Laguerre geometry. We first briefly review Laguerre geometry [1,8]: Via coordinates $\left(n_{0}, \mathbf{n}\right)$ with $\|\mathbf{n}\|=1$ for oriented planes, the set $\mathcal{P}$ of oriented planes is identified with the Blaschke cylinder $\mathbb{R} \times S^{2}$. We see that the set of oriented tangent planes of the sphere $(r, \mathbf{m})$ is given by $\left(\mathbb{R} \times S^{2}\right) \cap U$, where $U$ is a 3-dimensional subspace defined by the linear equation $x_{0}+x_{1} m_{1}+x_{2} m_{2}+x_{3} m_{3}=x_{0}+\langle\mathbf{x}, \mathbf{m}\rangle=r$. Laguerre transformations, which are the projective automorphisms of the Blaschke cylinder, map 3-dimensional subspaces not parallel to $(1,0,0,0)$ to subspaces which have the same property. It follows that being tangent to a common sphere is a Laguerre invariant of a $k$-tuple of oriented planes. So is being tangent to two spheres. If a $k$-tuple of oriented planes is tangent to two spheres of different radius, there is also a point contained in all of them. This last property however is not a Laguerre invariant. These considerations lead to the following theorem:

Theorem 12 For any conical mesh $\mathbf{v}: \mathbb{Z}^{2} \rightarrow \mathbb{R}^{3}$, we consider the face planes $F_{i j}$ and the corresponding coordinate vectors $\mathbf{f}_{i j} \in \mathbb{R} \times S^{2}$. Then $\mathbf{f}: \mathbb{Z}^{2} \rightarrow \mathbb{R}^{4}$ is a planar quad mesh which takes values in the Blaschke cylinder and whose 

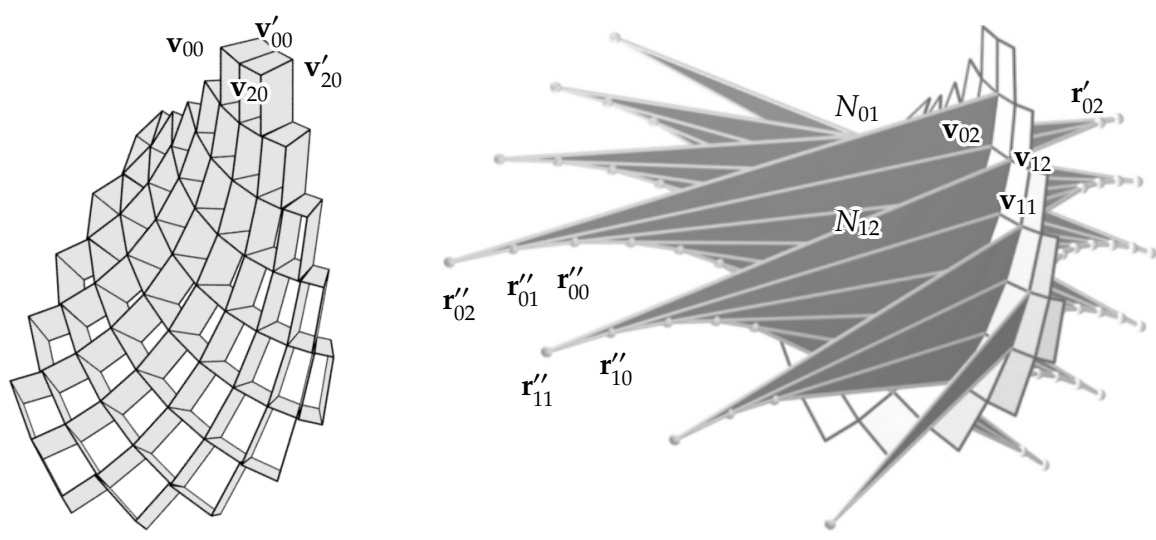

Fig. 5 Left: A conical mesh $\mathbf{v}$ with a face offset $\mathbf{v}^{\prime}$ and discrete normals which connect vertices $\mathbf{v}_{i j}$ with $\mathbf{v}_{i j}^{\prime}$. Right: The discrete developables consisting of discrete surface normals along the grid polylines of a conical mesh $\mathbf{v}$, together with the two focal meshes $\mathbf{r}^{\prime}$ and $\mathbf{r}^{\prime \prime}$.

face planes are not parallel to $\mathbf{e}_{0}=(1,0,0,0)$. Conversely, any planar quad mesh $\mathbf{f}: \mathbb{Z}^{2} \rightarrow \mathbb{R} \times S^{2}$ with this property arises in this way (if vertices are allowed to be at infinity). The conical property of meshes is a Laguerre geometry invariant.

Proof Only one thing remains to be shown. We start with a PQ mesh $\mathrm{f}$ in the Blaschke cylinder and construct a conical mesh $\mathbf{l}$ from it. An elementary quadrilateral $\mathbf{f}_{i j}, \mathbf{f}_{i+1, j}, \mathbf{f}_{i+1, j+1}, \mathbf{f}_{i, j+1}$ lies in a 2-dimensional subspace $V$ with $V$ not parallel to $\mathbf{e}_{0}$. We can choose two 3-dimensional affine subspaces $U, U^{\prime}$ with $U \cap U^{\prime}=V$ and neither $U, U^{\prime}$ parallel to $\mathbf{e}_{0}$. It follows that the planes corresponding to these four points of the Blaschke cylinder are tangent to two spheres, i.e., are incident with a common vertex, possibly at infinity. Therefore the oriented planes corresponding to $\mathbf{f}_{i j}$ occur as face planes of a mesh, which is conical by construction.

The geometries of Möbius and Laguerre can be treated in a unified way within the framework of Lie sphere geometry [8]. A discussion of conical and circular meshes from that perspective is given in [7].

\section{The discrete normals of circular and conical meshes}

From differential geometry we know that the principal curvature centers of a surface form the so-called focal surface, which usually consists of two sheets $[20,11,22]$. The ruled surface which consists of the surface normals along a principal curvature line is developable and touches these two sheets along a curve, whereas the singular curve of this developable surface is contained in the other focal sheet. Because we have two families of principal curvature lines, each sheet is covered by both contact curves and singular curves. They constitute a conjugate network. All these facts have discrete analogues for circular and conical meshes. 


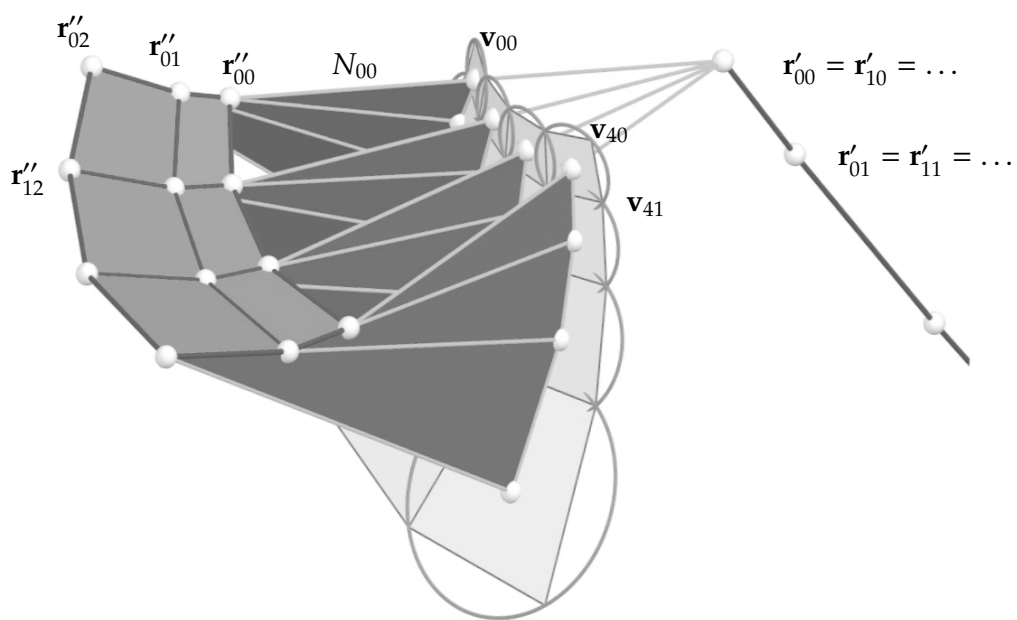

Fig. 6 A circular mesh $\mathbf{v}$ with discrete normal developables and focal meshes $\mathbf{r}^{\prime}$ and $\mathbf{r}^{\prime \prime}$. The mesh $\mathbf{r}^{\prime}$ here degenerates into a polygon, as $\mathbf{v}$ is a discrete canal surface.

\subsection{Normal developables and focal surfaces}

By definition, every vertex $\mathbf{v}_{i j}$ of a conical mesh is associated with a cone axis $N_{i j}$. We consider it as a discrete surface normal. Likewise, every face $F_{i j}$ of a circular mesh is associated with its circumcircle. We consider the axis $N_{i j}$ of this circle as a discrete surface normal. In both cases it is easy to see that the axes $N_{i j}$ and $N_{i+1, j}$ are coplanar, and so are the axes $N_{i j}$ and $N_{i, j+1}$ (see [17] for the conical case).

For fixed $i$, the axes $N_{i j}(j \in \mathbb{Z})$ constitute a discrete developable strip in the sense of [17]. The points $\mathbf{r}_{i j}^{\prime}=N_{i j} \cap N_{i+1, j}$ are considered discrete centers of curvature. Similarly we consider axes $N_{i j}$ with $j$ fixed and $i$ running, and get points $\mathbf{r}_{i j}^{\prime \prime}=N_{i j} \cap N_{i, j+1}$. We call the two meshes $\mathbf{r}^{\prime}, \mathbf{r}^{\prime \prime}$ the two focal meshes of the mesh we started with. This is illustrated by Figures 5 and 6 .

Proposition 13 The two focal meshes of a conical or circular mesh are PQ meshes (possibly degenerate).

Proof The points $\mathbf{r}_{i j}^{\prime}, \mathbf{r}_{i-1, j}^{\prime} \in N_{i j}$ and $\mathbf{r}_{i, j-1}^{\prime}, \mathbf{r}_{i-1, j-1}^{\prime} \in N_{i, j-1}$ are distributed on two coplanar lines, so they are co-planar. This shows that the elementary quadrilaterals of the mesh $\mathbf{r}^{\prime}$ are planar. A similar argument shows the same for the mesh $\mathbf{r}^{\prime \prime}$.

Analogous to the smooth setting, singularities of face offset meshes occur at the focal meshes.

A circular mesh $\mathbf{m}: \mathbb{Z}^{2} \rightarrow \mathbb{R}^{3}$ has more than just one way of defining a system of lines 'orthogonal' to the mesh. For any parallel mesh $\mathbf{m}^{\prime}$ of constant vertex distance we can consider the lines $L_{i j}$ spanned by the points $\mathbf{m}_{i j}$ and $\mathbf{m}_{i j}^{\prime}$ as discrete normals. It is interesting that this system of lines has properties similar to the previously defined discrete normals $N_{i j}$. 
Theorem 14 Consider a circular mesh $\mathbf{m}$ and a vertex offset $\mathbf{m}^{\prime}$ at nonzero distance according to Theorem 8 . The rows and the columns of the system of lines $L_{i j}=\mathbf{m}_{i j} \vee \mathbf{m}_{i j}^{\prime}$ form discrete developable surfaces.

The singular polygons of column developables $\left(L_{i j}\right)_{i \in \mathbb{Z}}$ have vertices $\mathbf{s}_{i j}^{\prime}=L_{i j} \cap$ $L_{i+1, j}$. Similarly, the row developables have vertices $\mathbf{s}_{i j}^{\prime \prime}=L_{i j} \cap L_{i, j+1}$. The discrete focal meshes $\mathbf{s}^{\prime}$ and $\mathbf{s}^{\prime \prime}$ of the system of lines $L_{i j}$ are planar quadrilateral meshes.

Proof As in the proof of Theorem 8, we consider the mesh $\widetilde{\mathbf{m}}: \mathbb{Z}^{2} \rightarrow S^{2}$ defined by $\widetilde{\mathbf{m}}=\left(\mathbf{m}^{\prime}-\mathbf{m}\right) / d$, which is parallel to $\mathbf{m}$. Parallelity implies that $\left\{\widetilde{\mathbf{m}}_{i j}, \widetilde{\mathbf{m}}_{i+1, j}, \mathbf{m}_{i+1, j}-\mathbf{m}_{i j}\right\}$ is linearly dependent. Therefore the lines $L_{i j}, L_{i+1, j}$ are co-planar, and the lines $L_{i j}$ form discrete developables along the rows in the mesh. The argument for columns is analogous.

The argument why the meshes $\mathbf{s}^{\prime}$ and $\mathbf{s}^{\prime \prime}$ are planar is exactly the same as in the proof of Proposition 13.

\subsection{The relation between circular and conical meshes}

There is some formal duality between circular and conical meshes. However, the relation of these two types of meshes is much closer: from a mesh of one type we can easily construct meshes of the other type, such that both meshes represent the same underlying surface. In this subsection, $\mathbf{m}: \mathbb{Z}^{2} \rightarrow \mathbb{R}^{3}$ will always be a circular mesh and $\mathbf{1}: \mathbb{Z}^{2} \rightarrow \mathbb{R}^{3}$ will be a conical mesh. This is easy to memorize, since conical meshes belong to Laguerre geometry, and circular meshes to Möbius geometry.

Theorem 15 For each conical mesh $\mathbf{1}: \mathbb{Z}^{2} \rightarrow \mathbb{R}^{3}$ with face planes $F_{i j}$ there is a two-parameter family of circular meshes $\mathbf{m}: \mathbb{Z}^{2} \rightarrow \mathbb{R}^{3}$ such that $\mathbf{m}_{i j} \in F_{i j}$. The vertices of $\mathbf{m}$ lie symmetric with respect to the edges of $\mathbf{1}$. Cone axes of the mesh $\mathbf{1}$ coincide with circle axes of the mesh $\mathbf{m}$.

Proof We start with a conical mesh 1 and choose a vertex, say $\mathbf{m}_{00}$, in the face $F_{00}$. We inductively construct the remaining points $\mathbf{m}_{i j}$ by the condition that vertices of the circular mesh contained in adjacent faces of the conical mesh lie symmetric with respect to the edge which these faces have in common (see Fig. 7, left) In order to show that this construction is consistent, we have to show that successive reflection of the point $\mathbf{m}_{i j}$ in the edges emanating from the vertex $\mathbf{l}_{i j}$ eventually leads to $\mathbf{m}_{i j}$ again. Here 'reflection' means reflection with respect to the intrinsic geometry of the mesh. We could also say that we use reflection in the bisector plane of the faces which contain the new vertices in question - this bisector contains the common edge of these faces, and also the cone axis associated with the vertex $\mathbf{l}_{i j}$. Fig. 7 , right explains why this is the case. First, the distance from $\mathbf{l}_{i j}$ is not affected by reflection, and both the original point and the result of reflection lie in a common plane orthogonal to the cone axes at the point $\mathbf{l}_{i j}$. Second, consider the angles $\omega_{1}, \ldots, \omega_{4}$ successively enclosed by the edges emanating from $\mathbf{1}_{i j}$ according to Proposition 1, and consider the angle $\alpha=\varangle\left(\mathbf{1}_{i+1, j} \mathbf{1}_{i, j} \mathbf{m}_{i j}\right)$. The angles $\beta, \gamma, \delta$ indicated in the figure have values $\beta=\omega_{1}-\alpha, \gamma=\omega_{2}-\beta$, and 


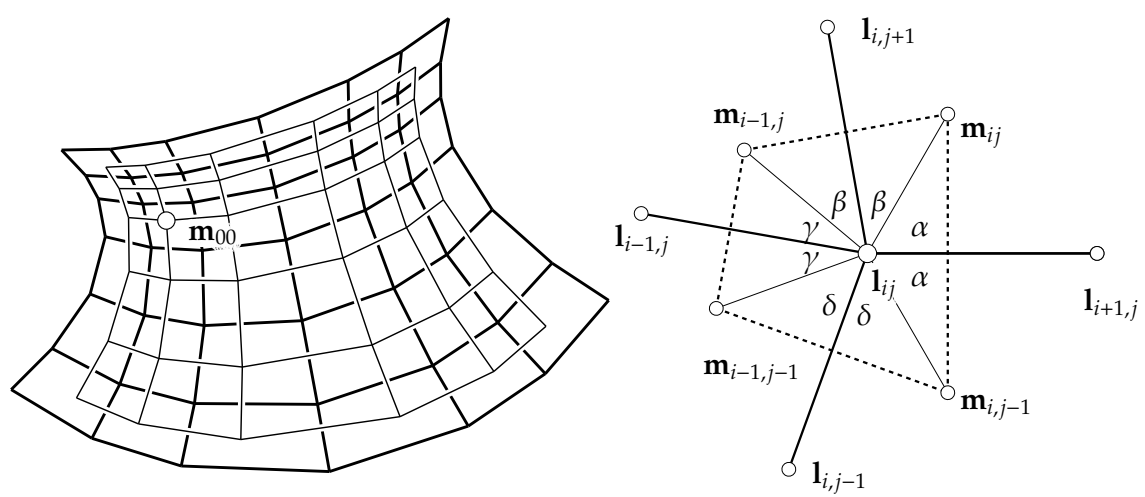

Fig. 7 Left: Construction of a circular mesh (thin lines) from a conical mesh (bold lines) by successive reflection of a vertex $\mathbf{m}_{00}$ in the edges of the conical mesh. Right: Top view in the direction of the cone axis at $\mathbf{l}_{i j}$.

$\delta=\omega_{3}-\gamma$. Consistency means that $\alpha$ must equal $\omega_{4}-\delta$. Expanding the definitions of $\beta, \gamma$, and $\delta$ and using the equality $\omega_{1}+\omega_{3}=\omega_{2}+\omega_{4}$ shows that this is indeed the case. The statement about the axes is obvious.

Theorem $\mathbf{1 6}$ For any conical mesh $\mathbf{1}$, and any given vertex $\mathbf{m}_{00}$, there is a unique circular mesh $\mathbf{m}$ such that the cone axes of $\mathbf{1}$ coincide with the circle axes of $\mathbf{m}$. If $\mathbf{m}_{00}$ happens to lie in the face plane $F_{00}$ of the conical mesh $\mathbf{1}$, this mesh $\mathbf{m}$ is the one constructed by Theorem 15, otherwise the vertices of $\mathbf{m}$ are contained in the face planes of a face offset of 1 .

All meshes $\mathbf{m}$ constructed in this way are parallel to each other.

Proof By replacing 1 by a face offset mesh we can without loss of generality assume that $\mathbf{m}_{00} \in F_{00}$. Neighbouring vertices $\mathbf{m}_{i j}$ and $\mathbf{m}_{i, j-1}$ are at constant distance from cone axes at $\mathbf{l}_{i j}$ and $\mathbf{l}_{i+1, j}$. This implies that they lie symmetric with respect to the edge $\mathbf{l}_{i j} \mathbf{1}_{i+1, j}$. For vertices $\mathbf{m}_{i j}$ and $\mathbf{m}_{i-1, j}$ we use a similar argument. This shows the symmetry which is used in the proof of Theorem 15 for constructing the mesh $\mathbf{m}$.

As edges of $\mathbf{m}$ are orthogonal both to the corresponding edges of 1 and the adjacent cone axes of $\mathbf{1}$, all meshes $\mathbf{m}$ are parallel to each other.

Corollary 17 The circular mesh constructed from a conical mesh according to Theorem 15 has the same discrete normals as the original mesh.

Remark 18 When constructing a face offset $\mathbf{1}^{(r)}$ of a conical mesh, vertices move along their cone axes. The axes remain discrete normals (cone axes) also for the offsets. Now consider the construction of Theorem 15 of a circular mesh whose vertices lie in the faces of a given conical mesh $\mathbf{1}$. We start with a vertex $\mathbf{m}_{00}$, which is chosen arbitrarily in the face $F_{00}$ of the mesh 1 . We also want to construct a circular mesh $\mathbf{m}^{\prime}$ associated with the offset $\mathbf{1}^{(r)}$. When we choose $\mathbf{m}_{00}^{\prime}$ such that $\mathbf{m}_{00}^{\prime}-\mathbf{m}_{00}$ is orthogonal to the face $F_{00}$, then the reflections employed in the proof of Theorem 15 which successively generate the mesh $\mathbf{m}$, will at the same time generate the mesh $\mathbf{m}^{\prime}$. Obviously, $\mathbf{m}^{\prime}$ is a vertex offset of $\mathbf{m}$. It follows that the construction given in Theorem 15 is offset-invariant. 
In view of Theorem 15 it is not surprising that we can also start with a circular mesh $\mathbf{m}$ and obtain a two-parameter family of conical meshes $\mathbf{1}$ which are tied to it in exactly the same relationship. The construction is in some sense dual to the one described above.

Theorem 19 For each circular mesh $\mathbf{m}: \mathbb{Z}^{2} \rightarrow \mathbb{R}^{2}$ there is a two-parameter family of conical meshes $\mathbf{1}$ with face planes $F_{i j}$ such that $\mathbf{m}_{i j} \in F_{i j}$. The planes $F_{i j}$ lie symmetric with respect to the edges of $\mathbf{m}$. Cone axes of the mesh $\mathbf{1}$ coincide with the circle axes of the mesh $\mathbf{m}$.

Proof We choose a face plane, say $F_{00}$, of the conical mesh 1 we want to construct. The only condition is that $\mathbf{m}_{00} \in F_{00}$. The other planes are constructed inductively by the requirement that the planes associated with adjacent vertices of the given circular mesh are symmetric with respect to the reflection which exchanges those two vertices. E.g., we construct $F_{i+1, j}$ by reflecting $F_{i j}$ in the symmetry plane of the vertices $\mathbf{m}_{i j}$ and $\mathbf{m}_{i+1, j}$. For consistency, we have to show that when traversing an elementary quadrilateral and constructing the planes associated with its vertices, after four reflections we get the plane we started with. This follows from the fact that the composition of the four reflections in the symmetry planes of the edges of a quadrilateral which is inscribed in a circle is a rotation by 360 degrees about the axis of that circle. Neighbouring faces (which are related by reflection) intersect in a line (contained in the symmetry plane of that reflection) which intersects the axis of the circumcircle. Further reflection does not affect this point, which shows that the newly constructed planes actually define edges which meet in the circle axes of the original mesh $\mathbf{m}$. This shows that 1 can be constructed, and that its discrete normals coincide with the discrete normals of the mesh $\mathbf{m}$.

Corollary 20 The constructions of Theorems 15 and 19 are inverse to each other.

Remark 21 The constructions of circular and conical meshes in Theorems 15 and 19 guarantee that a vertex $\mathbf{m}_{i j}$ of the circular mesh lies in the face plane $F_{i j}$ of the conical mesh. However in general we cannot expect that the vertices $\mathbf{m}_{i j}$ are contained in the actual faces of $\mathbf{1}$, which usually would be desirable for applications in geometric modeling.

The freedom we have in constructing a circular mesh associated with a conical mesh (pick one vertex, the rest is unique) is exactly the same freedom we have in constructing a parallel spherical mesh to a given circular mesh (again, pick one vertex, the rest is unique). This is no coincidence, as shown by Proposition 24 below.

\subsection{Polar duals of meshes}

There is the general notion of dual meshes where the vertices of the first mesh are in 1-1 correspondence with the faces of the second one, and vice versa. A duality with special geometric properties is the polar dual, which in this paper is used only for the case that the vertices of one mesh are contained in the unit sphere. 
Definition $22 P Q$ meshes $\widetilde{\mathbf{m}}$ and $\widetilde{\mathbf{l}}$ are called polar duals of each other, if the vertices of $\widetilde{\mathbf{m}}$ are contained in the unit sphere $S^{2}$, and the faces $\widetilde{F}_{i j}$ of the mesh $\widetilde{\mathbf{1}}$ are the polar duals of the vertices $\widetilde{\mathbf{m}}_{i j}$ (i.e., $\widetilde{F}_{i j}$ is the tangent plane of $S^{2}$ in the point $\widetilde{\mathbf{m}}_{i j}$ ).

Lemma 23 If $\widetilde{\mathbf{m}}: \mathbb{Z}^{2} \rightarrow S^{2}$ is a PQ mesh, then it has a polar dual, which is a conical mesh. Conversely, a conical mesh circumscribed to the unit sphere has a polar dual $\widetilde{\mathbf{m}}: \mathbb{Z}^{2} \rightarrow S^{2}$.

Proof Polarity maps co-planar vertices to planes which have a point in common. Points of $S^{2}$ are mapped to tangent planes of $S^{2}$, and vice versa.

The following result shows a construction of the family of conical meshes associated with a circular mesh according to Theorem 19. We establish a direct correspondence between associated conical meshes and associated parallel meshes in the unit sphere.

Proposition 24 Consider a circular mesh $\mathbf{m}$ and a conical mesh $\mathbf{1}$ such that the vertices of $\mathbf{m}$ lie in the faces of $\mathbf{1}$ (e.g. $\mathbf{m}$ is constructed from $\mathbf{1}$ via Theorem 15 , or $\mathbf{1}$ is constructed from $\mathbf{m}$ via Theorem 19). Further, consider meshes $\widetilde{\mathbf{m}}$ parallel to $\mathbf{m}$ and inscribed to the unit sphere, and $\widetilde{\mathbf{1}}$ parallel to $\mathbf{1}$ circumscribed to the unit sphere. Then $\widetilde{\mathbf{1}}$ and $\widetilde{\mathbf{m}}$ are polar duals of each other, and further, they are in the relation described by Theorems 15 and 19, i.e., vertices of $\widetilde{\mathbf{m}}$ lie in faces of $\widetilde{\mathbf{1}}$.

It follows that we could construct $\mathbf{1}$ from $\mathbf{m}$ by first constructing $\widetilde{\mathbf{m}}$, then find $\widetilde{\mathbf{1}}$ by polar duality, and translate the face planes of $\mathbf{1}$ through the vertices of $\mathbf{m}$.

Proof As the constructions of Theorems 15 and 19 are inverse to each other, we may for the purposes of this proof consider $\mathbf{m}$ to be constructed from 1 by successive reflection of the seed vertex $\mathbf{m}_{00}$, as described by Theorem 15. We are going to verify that also $\widetilde{\mathbf{m}}$ and $\widetilde{\mathbf{l}}$ are in that relation. We first consider the faces $\widetilde{F}_{i j}$ of $\widetilde{\mathbf{l}}$ and note the parallelities of edges $\widetilde{\mathbf{m}}_{00} \widetilde{\mathbf{m}}_{01} \| \mathbf{m}_{00} \mathbf{m}_{01}$ and faces $F_{i j} \| \widetilde{F}_{i j}$. This implies that $\widetilde{\mathbf{m}}_{01}$ arises from $\widetilde{\mathbf{m}}_{00}$ by applying the reflection $\widetilde{\sigma}_{00,01}$ in the bisector plane of $\widetilde{F}_{00}, \widetilde{F}_{01}$, simply because the same is true for vertices $\mathbf{m}_{00}, \mathbf{m}_{01}$ and faces $F_{00}, F_{01}$. Recall that $\widetilde{\mathbf{m}}_{00}$ has been chosen as the point $\widetilde{F}_{00} \cap S^{2}$ by construction, and that all planes $\widetilde{F}_{i j}$ are tangent planes of $S^{2}$. Thus, $\widetilde{\sigma}_{00,01}$ maps $S^{2}$ to $S^{2}$ and $\widetilde{F}_{00}$ to $\widetilde{F}_{01}$, which implies that $\widetilde{\mathbf{m}}_{01}=S^{2} \cap \widetilde{F}_{01}$, i.e., $\widetilde{\mathbf{m}}_{01}$ is the unit normal vector of planes $\widetilde{F}_{01}$ and $F_{01}$. This is exactly the condition that the plane $\widetilde{F}_{i j}$ is the polar dual of the vertex $\mathbf{m}_{i j}$, in the special case $(i, j)=(0,1)$. For $(i, j)=(0,0)$ this is true by construction.

It is clear how to continue this argument until by induction for all indices $(i, j)$ we have shown that the relation between $\widetilde{\mathbf{l}}$ and $\widetilde{\mathbf{m}}$ is as claimed, and the vertex $\widetilde{\mathbf{m}}_{i j}$ is the unit normal vector of both planes $\widetilde{F}_{i j}$ and $F_{i j}$.

Remark 25 The construction of a conical mesh which represents a discrete minimal surface in [25] by translating the tangent planes of an isothermic mesh $\widetilde{\mathbf{v}}: \mathbb{Z}^{2} \rightarrow S^{2}$ through the vertices of the discrete Christoffel dual mesh $\mathbf{v}$ is a special case of Proposition 24. 
5.4 Integrable distributions and normal congruences

For any 2-parameter family of straight lines $N(u, v)$ which locally are a fibration we can ask the question if these lines occur as the surface normals of a smooth surface $f(u, v)$ (in which case they are the surface normals of all offset surfaces as well). This is the case if and only if the distribution of all tangent planes $(p, \epsilon(p))$ with $p \in N(u, v)$ and $\epsilon \perp N(u, v)$ is integrable. The system of tangent planes orthogonal to the discrete normals of either a circular or conical mesh is an integrable distribution in a discrete sense:

Corollary 26 If the system of discrete normals (the discrete normal congruence) of a conical mesh or of a circular mesh is given, then for any choice of a vertex $\mathbf{m}_{00}$ there is a circular mesh $\mathbf{m}$ which has the given lines as discrete normals.

Proof If the normals of a conical mesh are given, the existence of $\mathbf{m}$ follows from Theorem 16. If the normals of a circular mesh are given, this is also true, because there exists a conical mesh with the same normals.

\section{Meshes which are both circular and conical}

Given the close relation between circular and conical meshes, it is natural to study those meshes which are both circular and conical. This means that we have to find all meshes which are parallel to a mesh $\widetilde{\mathbf{m}}$ whose vertices lie in the unit sphere $S^{2}$ and at the same time are parallel to a possibly different mesh $\widetilde{\mathbf{1}}$ whose face planes are tangent to $S^{2}$. As $\widetilde{\mathbf{m}}$ and 1 then are parallel meshes, and both circularity and conicality is invariant under mesh parallelism, it is clearly sufficient to construct a conical mesh $\widetilde{\mathbf{m}}: \mathbb{Z}^{2} \rightarrow S^{2}$ (which then is circular, being a PQ mesh in $S^{2}$ ). Such a mesh can be constructed from Cauchy-like data: we may prescribe two adjacent row polygons $\widetilde{\mathbf{m}}_{i 0}$ and $\widetilde{\mathbf{m}}_{i 1}$ and a column polygon $\widetilde{\mathbf{m}}_{0 j}$. From there we reconstruct $\widetilde{\mathbf{m}}$ by enforcing the conical condition at each vertex.

We are going to investigate an interesting subfamily of such meshes. First we discuss meshes in the unit sphere.

\subsection{Constant radius meshes and rhombic meshes in the unit sphere}

In this subsection we consider circular meshes in the sphere whose circumcircles have constant radius (SCR meshes), and collect some of their properties. It will turn out (see the next subsection) that they are closely related to the problem of finding meshes which have parallel meshes which are both face offsets and vertex offsets.

We assume now that $\widetilde{\mathbf{m}}: \mathbb{Z}^{2} \rightarrow S^{2}$ is an SCR mesh. For each face $F_{i j}$, which carries an elementary quadrilateral, we consider the circumcircle $\widetilde{C}_{i j}$ and its spherical center $\widetilde{\mathbf{c}}_{i j}$. For all $i, j$ we connect the point $\widetilde{\mathbf{c}}_{i j}$ with the vertices $\widetilde{\mathbf{m}}_{i j}, \widetilde{\mathbf{m}}_{i+1, j}, \widetilde{\mathbf{m}}_{i+1, j+1}, \widetilde{\mathbf{m}}_{i, j+1}$. Thus we obtain a rhombic mesh $\mathbf{r}: \mathbb{Z}^{2} \rightarrow S^{2}$ (see Fig. 8, left), whose vertex set is the union of vertex sets of 


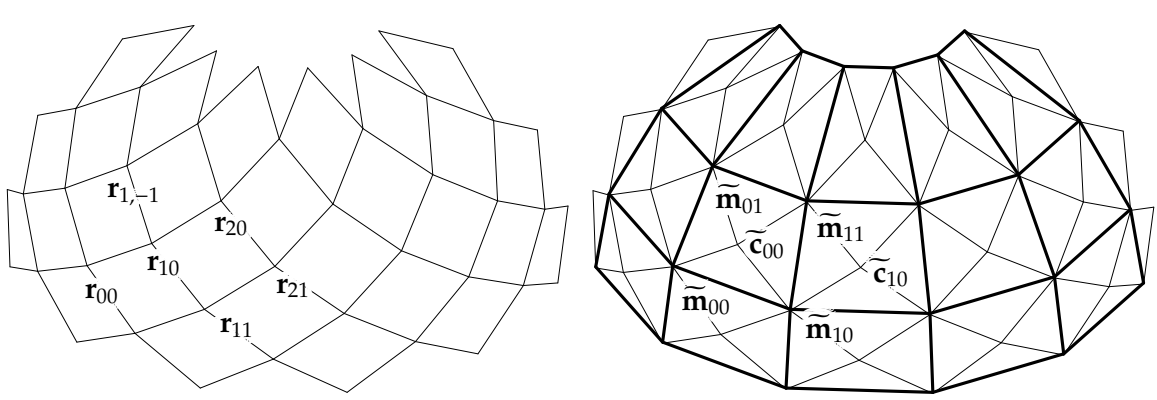

Fig. 8 Rhombic mesh $\mathbf{r}: \mathbb{Z}^{2} \rightarrow S^{2}$ (left) and its two diagonal meshes $\widetilde{\mathbf{m}}$ and $\widetilde{\mathbf{c}}$ (right). Any mesh $\mathbf{m}$ parallel to $\widetilde{\mathbf{m}}$ is both circular and conical, and has offset meshes which lie at both constant face distance and constant vertex distance from $\mathbf{m}$.

$\widetilde{\mathbf{m}}$ and $\widetilde{\mathbf{c}}$. All edges of $\mathbf{r}$ have constant length, i.e., all faces are non-planar rhomboids, and the two meshes $\widetilde{\mathbf{m}}$ and $\widetilde{\mathbf{c}}$ are the two diagonal meshes of $\mathbf{r}$ (see Fig. 8, right).

Proposition 27 Any SCR mesh $\widetilde{\mathbf{m}}: \mathbb{Z}^{2} \rightarrow S^{2}$ together with the mesh $\widetilde{\mathbf{c}}$ of its spherical centers of circumcircles arise as the two diagonal meshes of a rhombic mesh $\mathbf{r}$. For all rhombic meshes, the two diagonal meshes are SCR meshes, and they are in this relationship.

Proof The first part is the discussion above. As to the second statement, we start with a rhombic mesh $\mathbf{r}: \mathbb{Z}^{2} \rightarrow S^{2}$, and denote its two diagonal meshes by $\widetilde{\mathbf{m}}$ and $\widetilde{\mathbf{c}}$, where the indices are chosen such that the distances $\left\|\widetilde{\mathbf{c}}_{i j}-\widetilde{\mathbf{m}}_{i j}\right\|$, $\left\|\widetilde{\mathbf{c}}_{i j}-\widetilde{\mathbf{m}}_{i+1, j}\right\|,\left\|\widetilde{\mathbf{c}}_{i j}-\widetilde{\mathbf{m}}_{i+1, j+1}\right\|$, and $\left\|\widetilde{\mathbf{c}}_{i j}-\widetilde{\mathbf{m}}_{i, j+1}\right\|$, which are measured along edges of $\mathbf{r}$, are constant. This means that all points of an elementary quadrilateral of $\widetilde{\mathbf{m}}$ are at constant distance from $\widetilde{\mathbf{c}}_{i j}$, i.e., the elementary quadrilateral is planar, and has a circumcircle whose radius does not depend on $i, j$.

Proposition 28 Any SCR mesh $\widetilde{\mathbf{m}}$ of radius $r$ is also conical of constant cone opening angle $\phi$, with $\cos (\phi / 2)=r$. The mesh $\widetilde{\mathbf{c}}$ of spherical centers associated with $\widetilde{\mathbf{m}}$ has the same property. The polar dual of $\widetilde{\mathbf{m}}$ equals $\frac{1}{\sqrt{1-r^{2}}} \cdot \widetilde{\mathbf{c}}$, and vice versa.

Proof The statement about polar duals is elementary: the tangent planes in the points of a circle in $S^{2}$ having spherical center $\widetilde{\mathbf{c}}$ and Euclidean radius $r$ meet in the point $1 / \sqrt{1-r^{2}} \mathbf{c}$. As to the first statement, assume that points lie in a circle of radius $r$ contained in $S^{2}$. Polar duality maps these points to planes tangent to a cone of revolution, whose opening angle obeys $\cos (\phi / 2)=r$. This shows the first statement. The fact that $\mathbf{c}$ has the same properties follows from the fact that both $\widetilde{\mathbf{m}}$ and $\widetilde{\mathbf{c}}$ arise in the same manner as diagonal mesh of a rhombic mesh.

6.2 Meshes which possess face+vertex offsets

A mesh $\mathbf{m}: \mathbb{Z}^{2} \rightarrow \mathbb{R}^{3}$ which is both conical and circular possesses a parallel mesh $\mathbf{m}^{\prime}$ at constant face distance $D \neq 0$ (a face offset) and also a parallel 

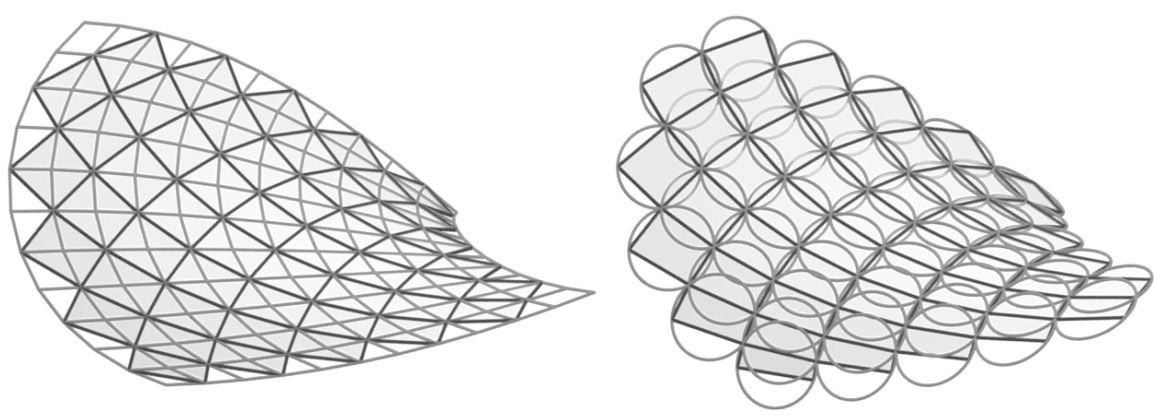

Fig. 9 A discrete surface of negative Gaussian curvature in the shape of a circular mesh (right), which arises as the diagonal mesh of a rhombic mesh (left). It is circular of constant radius, and also conical of constant opening angle. The mesh is also shown by Fig. 5, together with a face+vertex offset.

mesh $\mathbf{m}^{\prime \prime}$ at constant vertex distance $d \neq 0$ (a vertex offset). The most interesting case here is that there is a mesh with both properties, i.e., where we can choose $\mathbf{m}^{\prime}=\mathbf{m}^{\prime \prime}$ :

Proposition 29 If a mesh $\mathbf{m}: \mathbb{Z}^{2} \rightarrow \mathbb{R}^{3}$ has a parallel mesh $\mathbf{m}^{\prime}$ which is a face offset for distance $D$ and vertex offset at distance d, then it has a parallel SCR mesh $\widetilde{\mathbf{m}}: \mathbb{Z}^{2} \rightarrow S^{2}$ with circle radius $r=\sqrt{1-D^{2} / d^{2}}$. Conversely, any mesh parallel to an SCR mesh $\widetilde{\mathbf{m}}$ has offset meshes which are not only at constant face distance, but also at constant vertex distance. Such meshes are also conical, with constant cone opening angle.

Proof By our assumptions, the mesh $\widetilde{\mathbf{m}}:=\frac{1}{d}\left(\mathbf{m}^{\prime}-\mathbf{m}\right)$ has vertices in the unit sphere, and its faces are at distance $D / d$ from the origin o. Obviously, $D / d<1$. Consider the face planes $\widetilde{F}_{i j}$ of $\widetilde{\mathbf{m}}$ and the circles $\widetilde{C}_{i j}=S^{2} \cap \widetilde{F}_{i j}$. Since $\operatorname{dist}\left(\widetilde{F}_{i j}, \mathbf{o}\right)=D / d=$ const, the radius of the circles $\widetilde{C}_{i j}$ is as stated above.

Conversely we may start with $\widetilde{\mathbf{m}}$ and consider any mesh $\mathbf{m}$ parallel to $\widetilde{\mathbf{m}}$. The mesh $\mathbf{m}^{\prime}=\mathbf{m}+\delta \widetilde{\mathbf{m}}$ is at vertex distance $\delta$ and, by construction, at face distance $\delta \frac{D}{d}$.

The statement about the cone opening angle follows directly from Proposition 28 via parallelity.

We combine previous results on the relationship of conical meshes and circular meshes to this case of meshes with both properties:

Theorem 30 A mesh $\mathbf{m}$ which has offset meshes at both constant face and vertex distance is circular and conical of constant cone opening angle. There is another mesh $\mathbf{c}$ with the same properties and the same cone opening angle, whose face planes pass through the vertices of $\mathbf{m}$, and such that there is a rhombic mesh $\mathbf{r}: \mathbb{Z}^{2} \rightarrow S^{2}$ whose diagonal meshes $\widetilde{\mathbf{m}}$ and $\widetilde{\mathbf{c}}$ are parallel to $\mathbf{m}$ and $\mathbf{c}$, respectively.

Proof We construct $\mathbf{m}$ and the SCR mesh $\widetilde{\mathbf{m}}$ parallel to $\mathbf{m}$ according to Proposition 29, so that there is a rhombic mesh $\mathbf{r}$ whose diagonal mesh is $\widetilde{\mathbf{m}}$. The other diagonal mesh $\widetilde{\mathbf{c}}$ is also an SCR mesh of the same cone opening 
angle. We now construct a conical mesh $\mathbf{c}$ associated with $\mathbf{m}$ according to the procedure described by Proposition 24, and we choose the mesh $\widetilde{\mathbf{m}}$ for that. Then $\mathbf{c}$ is parallel to the polar dual of $\widetilde{\mathbf{m}}$. As $\widetilde{\mathbf{c}}$ is a homothetical copy of that polar dual, $\mathbf{c}$ and $\widetilde{\mathbf{c}}$ are parallel. Now the properties of $\mathbf{c}$ stated in the theorem follow from Proposition 29 - neither of $\mathbf{m}, \mathbf{c}$ is distinguished before the other, as the two diagonal meshes of a given rhombic mesh are completely interchangeable.

\subsection{Discrete surfaces of constant negative Gaussian curvature}

There is one special instance of mesh pairs $\mathbf{m}, \mathbf{c}$ according to Theorem 30 which has to be mentioned. These meshes are diagonal meshes of a rhombic mesh with planar vertex stars. Such rhombic meshes have been studied by W. Wunderlich [27] as discrete surfaces with constant negative Gaussian curvature. The meshes shown by Figures 5 and 9 are of that type. It should be noted that in this special case, both meshes $\mathbf{m}$ and $\mathbf{c}$ have constant circumcircle radius. By construction, the vertices of $\mathbf{c}$ lie in the face planes of $\mathbf{m}$, and vice versa.

\section{Conclusion and future research}

We have studied conical meshes and circular meshes with an emphasis on discrete surface normals. We first considered parallel meshes inscribed or circumscribed to the unit sphere, and parallel meshes at constant face or vertex distance. Briefly we have related the geometry of circular and conical meshes to Möbius and Laguerre geometry. The discrete normals of a circular or conical mesh can be arranged in discrete developables analogous to the discrete developables associated with the principal curvature lines of a smooth surface. Their singular polygons form two focal surfaces. Such a construction is not only possible for the discrete normals, but also for the lines which connect a circular mesh with its parallel meshes at constant vertex distance.

The 2-parameter family of conical meshes associated with a circular mesh and vice versa are the topic of Section 5.2. The construction of meshes of the other type which belong to the same underlying surface makes use either of an inductive construction via reflections, or of the polar dual with respect to the unit sphere.

The last section deals with meshes which are both circular and conical, and in particular with meshes which have offsets, which are not only at constant face distance, but at the same time also at constant vertex distance. They turn out to be closely related to rhombic meshes in the sphere. A special case of such meshes is derived from rhombic meshes of constant negative Gaussian curvature.

There are several natural generalizations of the present work. We have studied meshes which possess face offsets and/or vertex offsets. For architectural applications the case of a parallel mesh where edges are at constant 
distance is particularly interesting. This means that there exists a Combescure transform whose edges are tangent to the unit sphere. Meshes with this property are Laguerre geometric counterparts of S-isothermic meshes [4]. They are the topic of a forthcoming publication; for initial results, see [21]. The systematic approach to offset properties via Combescure transforms tangent to unit balls carries over to Minkowski geometry and leads to various discretizations of the relative principal curvature lines [21].

\section{Acknowledgments}

The authors wish to express their thanks to A. Bobenko and Yu. Suris for communicating their unpublished work on circular and conical meshes from the Lie geometry viewpoint. This research has been supported by grants No. S9206-N12 and No. P19214-N18 of the Austrian Science Fund (FWF).

\section{References}

1. W. Blaschke. Vorlesungen über Differentialgeometrie, volume 3. Springer, 1929.

2. A. Bobenko. Discrete conformal maps and surfaces. In Symmetries and integrability of difference equations, volume 255 of London Math. Soc. Lecture Note Ser., pages 97-108. Cambridge Univ. Press, 1999.

3. A. Bobenko and U. Hertrich-Jeromin. Orthogonal nets and Clifford algebras. Tohoku Math. Publ., 20:7-22, 2001.

4. A. Bobenko, T. Hoffmann, and B. Springborn. Minimal surfaces from circle patterns: Geometry from combinatorics. Annals of Mathematics, 164:231-264, 2006.

5. A. Bobenko and U. Pinkall. Discrete isothermic surfaces. J. Reine Angew. Math., 475:187-208, 1996.

6. A. Bobenko and Yu. Suris. Discrete differential geometry. Consistency as integrability. preprint, http://arxiv.org/abs/math.DG/0504358, 2005.

7. Alexander Bobenko and Yu. Suris. On organizing principles of discrete differential geometry, geometry of spheres. preprint, http://arxiv.org/abs/math.DG/0608291, 2006.

8. T. Cecil. Lie Sphere Geometry. Springer, 1992.

9. J. Cieśliński, A. Doliwa, and P. M. Santini. The integrable discrete analogues of orthogonal coordinate systems are multi-dimensional circular lattices. Phys. Lett. A, 235:480-488, 1997.

10. M. Desbrun, E. Grinspun, P. Schröder, K. Polthier, et al. Discrete differential geometry. SIGGRAPH Course Notes, 2006. http://ddg.cs.columbia.edu.

11. M. do Carmo. Differential Geometry of Curves and Surfaces. Prentice-Hall, 1976.

12. A. Doliwa, S. V. Manakov, and P. M. Santini. $\bar{\partial}$-reductions of the multidimensional quadrilateral lattice. The multidimensional circular lattice. Comm. Math. Phys., 196:1-18, 1998.

13. A. Doliwa and P. M. Santini. Multidimensional quadrilateral lattices are integrable. Phys. Lett. A, 233(4-6):365-372, 1997.

14. A. Doliwa, P. M. Santini, and M. Mañas. Transformations of quadrilateral lattices. J. Math. Phys., 41:944-990, 2000.

15. U. Hertrich-Jeromin. Introduction to Möbius differential geometry, volume 300 of London Mathematical Society Lecture Note Series. Cambridge University Press, 2003. 
16. B. G. Konopelchenko and W. K. Schief. Three-dimensional integrable lattices in Euclidean spaces: conjugacy and orthogonality. R. Soc. Lond. Proc. Ser. A Math. Phys. Eng. Sci., 454:3075-3104, 1998.

17. Y. Liu, H. Pottmann, J. Wallner, Y. Yang, and W. Wang. Geometric modeling with conical meshes and developable surfaces. ACM Trans. Graphics, 25(3):681-689, 2006. Proc. SIGGRAPH 2006.

18. M. Mañas, A. Doliwa, and P. M. Santini. Darboux transformations for multidimensional quadrilateral lattices. I. Phys. Lett. A, 232:99-105, 1997.

19. R. R. Martin, J. de Pont, and T. J. Sharrock. Cyclide surfaces in computer aided design. In J. A. Gregory, editor, The mathematics of surfaces, pages 253-268. Clarendon Press, Oxford, 1986.

20. I. R. Porteous. Geometric Differentiation for the Intelligence of Curves and Surfaces. Cambridge Univ. Press, 1994.

21. H. Pottmann. Generalized principal meshes and their support structures. Geometry Preprint 158, Technische Universität Wien, 2006.

22. H. Pottmann and J. Wallner. Computational Line Geometry. Springer, 2001.

23. R. Sauer. Parallelogrammgitter als Modelle pseudosphärischer Flächen. Math. Zeitschr., 52:611-622, 1950.

24. R. Sauer. Differenzengeometrie. Springer, 1970.

25. J. Wallner and H. Pottmann. Infinitesimally flexible meshes and discrete minimal surfaces. Geometry Preprint 162, Technische Universität Wien. http://www.geometrie. tuwien.ac.at/wallner/cmin.pdf, 2006.

26. W. Wang, J. Wallner, and Y. Liu. An angle criterion for conical mesh vertices. Geometry Preprint 157, Technische Universität Wien. http://www.geometrie.tuwien. ac.at/ig/papers/tr157.pdf, 2006.

27. W. Wunderlich. Zur Differenzengeometrie der Flächen konstanter negativer Krümmung. Sitz. Öst. Ak. Wiss., 160:41-77, 1951. 